\title{
Modelling of High Torque Density Switched Reluctance Motors with Mutual Coupling
}

\author{
Mario Greule ${ }^{1)}$, Johannes Müller ${ }^{2)}$ and Martin Doppelbauer ${ }^{3)}$ \\ 1) 2) 3) Karlsruhe Institute of Technology, Karlsruhe, Germany, \\ e-mail: ${ }^{1)}$ Mario.Greule@kit.edu, ${ }^{2}$ Johannes.Mueller@student.kit.edu, ${ }^{3)}$ Martin.Doppelbauer@kit.edu
}

\begin{abstract}
In this paper a method is introduced which models the resulting motor torque and current dynamics directly from the flux linkages. With the introduced method to process the flux linkage characteristics it is sufficient to calculate half of an electrical period to receive all information for modelling the system's behaviour, even for both long and short flux path mutual coupling. The model is validated by $2 \mathrm{D}$ finite element analysis and verified by measurement results.
\end{abstract}

Keywords - switched reluctance motor, model, mutual coupling, eddy current losses, control

\section{INTRODUCTION}

The power density of switched reluctance motors can be increased by high speeds or by minimizing the yoke thickness. While the latter can lead to an increase of noise [1], this drawback can be acceptable in many applications, e.g. a starter-generator in a conventional car, which is masked by the combustion engine. For this application low noise emission is not that relevant, but a high power density and a low price is most important. These are properties which can be fulfilled by a SRM with minimized yoke thickness. To accurately simulate or control such a motor an improved SRM model is necessary which incorporates the mutual coupling between the motor phases.

The effect of mutual coupling has been discussed by several authors [2-5]. Especially in [2] the influence has been discussed in detail and was proven by experiment. It has been shown that mutual coupling and the consequent torque deterioration is dependent on the phase connection sequence [2, 3]. An alternating stator connection sequence of north and south poles leads to a better torque output than subsequent north or south poles.

While the voltage equation of the SRM has been modeled by several authors by using flux linkages, e.g. to realize sensorless control schemes $[4,6]$, there is no correct solution to model the resulting torque by flux linkages. There are some publications which model the resulting torque by self and mutual inductances [3, 7]. This is possible but more complicated than using flux linkages, which can be measured [2], to calculate the resulting torque. In [5] the resulting torque of mutual coupled flux paths is stored in look up tables, which has the disadvantage that the torque must be calculated by a reluctance model or finite element analysis (FEA) and cannot be measured on a real motor with sufficient accuracy.
In the following Section the dynamic model of the SRM with mutual coupled flux paths is theoretically derived by the theory of energy functionals. In Section III the theory is applied to a 3-phase SRM with the necessary data derived from a static finite element analysis. With only half an electrical period it is possible to describe the total dynamic behavior, both for pole commutation with short or long flux paths. The validation of the dynamic model is done in Section IV, by calculating the phase currents offline in a system simulation with Direct Instantaneous Torque Control (DITC) [8] and exporting them to a dynamic 2D finite element analysis. In Section $\mathrm{V}$ the model is verified by comparison of simulated and measured current profiles and the mean output torque at different operating points.

This paper is an extended version of [9] which verifies the SRM model and the FEA validation by measurement results.

\section{DERIVATION OF THE GENERALIZED MODEL}

To simulate or control an $n$-phase SRM the terminal voltage $\boldsymbol{u}=\left(u_{1}, u_{2}, \ldots, u_{n}\right)$, the input current $\boldsymbol{i}=$ $\left(i_{1}, i_{2}, \ldots, i_{n}\right)$, the torque generation $T$ and the motor speed $\Omega$ are of interest. The conversion between these system variables can be done by the theory of energy functionals. For electrical machines this theory is derived, for example, in [10] and applied to a permanent magnet machine in [11]. As depicted in Fig. 1, the electrical system and the mechanical system are coupled by an electromagnetic energy converter [10].

It is assumed that the energy converter has no dissipative components, which is true if all loss components, e.g. the resistive losses, the friction losses and even the iron losses, are neglected or modeled outside the coupling field in the electrical or mechanical system. Thus the internal currents and voltages $(\boldsymbol{i}, \boldsymbol{u})$ do not need to be the same as the terminal currents and voltages $\left(\boldsymbol{i}_{\mathrm{e}}, \boldsymbol{u}_{\mathrm{e}}\right)$. The same applies to the internal generalized mechanical force and velocity $(T, \Omega)$ and the external torque and angular velocity $\left(T_{\mathrm{e}}, \Omega_{\mathrm{e}}\right)$. Providing that the internal currents $\boldsymbol{i}=\left(i_{1}, i_{2}, \ldots, i_{n}\right)$ and the internal generalized force $T$ are independent, the energy $W_{\mathrm{m}}$ at the time $t$ which is stored by the magnetic field can be stated as

$$
\begin{aligned}
\mathrm{W}_{\mathrm{m}}(t) & =W_{\mathrm{m}}(0) \\
& +\int_{0}^{t}\left(\sum_{k=1}^{n} u_{k}(\tilde{t}) i_{k}(\tilde{t})-\Omega(\tilde{t}) T(\tilde{t})\right) \mathrm{d} \tilde{t}
\end{aligned}
$$




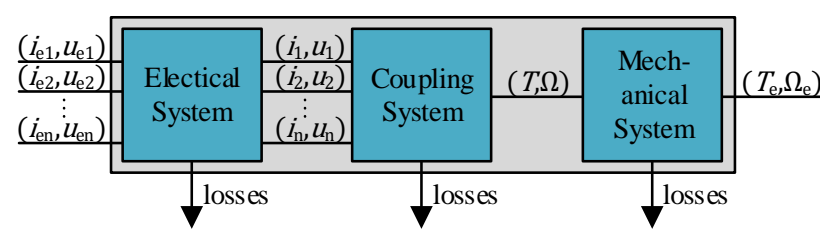

Fig. 1. System components of an electric motor, coupled by the system variables.

with the electrical power of each node $P_{\mathrm{el}, k}=u_{k} \cdot i_{k}$ and the mechanical power $P_{\text {mech }}=T \cdot \Omega$. Here the nomenclature is chosen that field energy is increased by (a positive) electrical power and decreased by mechanical power. As $u_{k}=\mathrm{d} \Psi_{\mathrm{k}} / \mathrm{d} t$ and $\Omega=\mathrm{d} \theta / \mathrm{d} t$, with the magnetic flux linkage $\boldsymbol{\Psi}=\left(\Psi_{1}, \Psi_{2}, \ldots, \Psi_{n}\right)$ and the angular motor angle $\theta$, equation (1) follows to

$$
\begin{aligned}
\mathrm{W}_{\mathrm{m}}(\theta, \boldsymbol{\Psi}) & =W_{\mathrm{m}}\left(\theta_{0}, \boldsymbol{\Psi}_{\mathbf{0}}\right)+\int_{\boldsymbol{\Psi}_{\mathbf{0}}}^{\boldsymbol{\Psi}} \sum_{k=1}^{n} i_{k}(\theta, \widetilde{\boldsymbol{\Psi}}) \mathrm{d} \widetilde{\Psi}_{k} \\
& -\int_{\theta_{0}}^{\theta} T(\tilde{\theta}, \boldsymbol{\Psi}) \mathrm{d} \tilde{\theta}
\end{aligned}
$$

Since it has been assumed that the inner magnetic field has no dissipative components the following condition holds true [12]:

$$
\frac{\partial \Psi_{j}}{\partial r_{k}}=\frac{\partial \Psi_{k}}{\partial r_{j}}
$$

with $j, k=1,2, \ldots, n+1$ and $j \neq k$. This means that the integral of (2) is path independent and so it can be rearranged to

$$
\begin{aligned}
\mathrm{W}_{\mathrm{m}}(\theta, \boldsymbol{\Psi}) & =W_{\mathrm{m}}\left(\theta_{0}, \boldsymbol{\Psi}_{\mathbf{0}}\right)+\int_{\boldsymbol{\Psi}_{\mathbf{0}}}^{\boldsymbol{\Psi}} \sum_{k=1}^{n} \frac{\partial \mathrm{W}_{\mathrm{m}}(\theta, \widetilde{\Psi})}{\partial \widetilde{\Psi}_{k}} \mathrm{~d} \widetilde{\Psi}_{k} \\
& -\int_{\theta_{0}}^{\theta} \frac{\partial \mathrm{W}_{\mathrm{m}}(\widetilde{\theta}, \boldsymbol{\Psi})}{\partial \widetilde{\theta}_{k}} \mathrm{~d} \tilde{\theta}
\end{aligned}
$$

By comparing the coefficients of (2) and (4) the current in dependence of the flux linkages and the torque can be derived. As it is more intuitive to describe the flux linkages in dependence of the phase currents the Legendre transformation

$$
W_{\mathrm{m}}^{*}(\theta, \boldsymbol{i})=\mathbf{\Psi}(\theta, \boldsymbol{i}) \cdot \boldsymbol{i}-W(\Psi(\theta, \boldsymbol{i}), \theta)
$$

is applied to yield the coenergy $W_{\mathrm{m}}^{*}$ with the independent variables phase currents $\boldsymbol{i}$ and motor angle $\theta$. As a result, equations (2) and (4) lead to (6) and (7):

$$
\begin{aligned}
W_{\mathrm{m}}^{*}(\theta, \boldsymbol{i}) & =W_{\mathrm{m}}^{*}\left(\theta_{0}, \boldsymbol{i}_{\mathbf{0}}\right) \\
& +\int_{\boldsymbol{\Psi}_{\mathbf{0}}}^{\boldsymbol{\Psi}} \sum_{k=1}^{n} \Psi_{k}(\theta, \tilde{\boldsymbol{\imath}}) \mathrm{d} \tilde{\imath}_{k}-\int_{\theta_{0}}^{\theta} T(\tilde{\theta}, \boldsymbol{i}) \mathrm{d} \tilde{\theta} \\
W_{\mathrm{m}}^{*}(\theta, \boldsymbol{i}) & =W_{\mathrm{m}}^{*}\left(\theta_{0}, \boldsymbol{i}_{\mathbf{0}}\right) \\
& +\int_{\boldsymbol{\Psi}_{\mathbf{0}}}^{\boldsymbol{\Psi}} \sum_{k=1}^{n} \frac{\partial W_{\mathrm{m}}^{*}(\theta, \tilde{\boldsymbol{\imath}})}{\partial \tilde{\imath}_{k}} \mathrm{~d} \tilde{\iota}_{k}-\int_{\theta_{0}}^{\theta} \frac{\partial W_{\mathrm{m}}^{*}(\widetilde{\theta}, \boldsymbol{i})}{\partial \widetilde{\theta}_{k}} \mathrm{~d} \tilde{\theta}
\end{aligned}
$$

From (6) and (7) the resulting torque can be derived by comparing the coefficients:

$$
T=\frac{\partial W_{\mathrm{m}}^{*}}{\partial \theta}
$$

Furthermore, by an appropriate choice of $\left(\theta_{0}, \boldsymbol{i}_{\mathbf{0}}\right)$ the term $W_{\mathrm{m}}^{*}\left(\theta_{0}, \boldsymbol{i}_{\mathbf{0}}\right)$ equals zero and with a fixed angle $\theta=$ $\theta_{0}$ the coenergy can be derived as follows:

$$
W_{\mathrm{m}}^{*}\left(\theta_{0}, \boldsymbol{i}\right)=\int_{i_{0}}^{i} \sum_{k=1}^{n} \Psi_{k}\left(\theta_{0}, \tilde{\boldsymbol{\imath}}\right) \mathrm{d} \tilde{l}_{k}
$$

This equation implies that the coenergy can be derived for a fixed angle by integrating the flux linkage over each phase current and summation of the results for each phase.

The magnetic flux linkage $\boldsymbol{\Psi}$ in dependence of the current $\boldsymbol{i}$ can be derived by a finite element analysis or by measurement from the general voltage equation:

$$
\boldsymbol{u}_{\mathrm{e}}=\boldsymbol{R} \boldsymbol{i}+\frac{\mathrm{d}}{\mathrm{d} t} \boldsymbol{\Psi}
$$

This implies that all electric and magnetic losses are known and can be modeled as a series resistance $\boldsymbol{R}$, which requires that the external currents $\boldsymbol{i}_{\mathrm{e}}$ equal the internal currents $\boldsymbol{i}$. This assumption is not generally valid and represents an approximation. A practical implementation to measure the flux linkages of each phase with mutual coupling can be found in [2].

The voltage equation in dependence of the state variables current and motor angle can be derived from (10) by partial differentiation:

$$
\boldsymbol{u}_{\mathrm{e}}=\boldsymbol{R} \boldsymbol{i}+\frac{\partial \Psi}{\partial \theta} \cdot \Omega+\sum_{k=1}^{n} \frac{\partial \Psi}{\partial i_{k}} \frac{\mathrm{d} i_{k}}{\mathrm{~d} t}
$$

with the angular velocity $\Omega=\partial \theta / \partial t$.

In summary the physical behavior of the $n$-phase SRM can be described by the torque equation (8) and the voltage equation (11) in dependency of the state variables, which are the phase currents $\boldsymbol{i}=\left(i_{1}, i_{2}, \ldots, i_{n}\right)$ and the motor angle $\theta$.

\section{APPLiCATION OF THE MOdEL to A 3-PHASE SRM}

This theory is applied to model the physical behavior of a three phase SRM. The model is implemented in Matlab Simscape, which allows non-causal modeling. Due to the boundary conditions the non-defined variables are determined, e.g. with a defined voltage input and a defined motor speed the resulting torque and input current are derived by the model. If the load torque is given as a boundary condition the resulting motor speed is determined in the simulation.

The physical behavior of each phase is modeled individually in dependence of all state variables $(\theta, \boldsymbol{i})=$ $\left(\theta, i_{1}, i_{2}, i_{3}\right)$. It is presumed that the current profiles of each phase are identical with an electrical phase shift of $120^{\circ}$. That is why only phase one is described here.

Applying (11) to a three phase SRM the voltage of phase one leads to

$$
\begin{aligned}
u_{1} & =R_{1} i_{1}+\frac{\partial \Psi_{1}\left(\theta, i_{1}, i_{2}, i_{3}\right)}{\partial \theta} \frac{\mathrm{d} \theta}{\mathrm{d} t} \\
& +\frac{\partial \Psi_{1}\left(\theta, i_{1}, i_{2}, i_{3}\right)}{\partial i_{1}} \frac{\mathrm{d} i_{1}}{\mathrm{~d} t}+\frac{\partial \Psi_{1}\left(\theta, i_{1}, i_{2}, i_{3}\right)}{\partial i_{2}} \frac{\mathrm{d} i_{2}}{\mathrm{~d} t} \\
& +\frac{\partial \Psi_{1}\left(\theta, i_{1}, i_{2}, i_{3}\right)}{\partial i_{3}} \frac{\mathrm{d} i_{3}}{\mathrm{~d} t}
\end{aligned}
$$

It can be seen that the voltage equation of phase one only depends on the flux linkage of phase one.

In contrast, the resulting torque of a mutual coupled SRM cannot be allocated to the individual phases as shown below. The resulting torque is derived by (8) with the coenergy given by (9). For a three phase SRM the coenergy is determined as follows: 


$$
\begin{aligned}
W_{\mathrm{m}}^{*}\left(\theta_{0}, \boldsymbol{i}\right) & =\int_{0}^{\boldsymbol{i}_{1}} \Psi_{1}\left(\theta_{0}, \tilde{l}_{1}, 0,0\right) \mathrm{d} \tilde{l}_{1} \\
& +\int_{0}^{\boldsymbol{i}_{2}} \Psi_{2}\left(\theta_{0}, i_{1}, \tilde{l}_{2}, 0\right) \mathrm{d} \tilde{l}_{2} \\
& +\int_{0}^{\boldsymbol{i}_{3}} \Psi_{3}\left(\theta_{0}, i_{1}, i_{2}, \tilde{l}_{3}\right) \mathrm{d} \tilde{l}_{3}
\end{aligned}
$$

Due to the path independence of the integral of (9) the coenergy can also be stated as (14) or (15).

$$
\begin{aligned}
W_{\mathrm{m}}^{*}\left(\theta_{0}, \boldsymbol{i}\right) & =\int_{0}^{\boldsymbol{i}_{2}} \Psi_{2}\left(\theta_{0}, 0, \tilde{\iota}_{2}, 0\right) \mathrm{d} \tilde{\iota}_{2} \\
& +\int_{0}^{\boldsymbol{i}_{1}} \Psi_{1}\left(\theta_{0}, \tilde{\iota}_{1}, i_{2}, 0\right) \mathrm{d} \tilde{l}_{1} \\
& +\int_{0}^{\boldsymbol{i}_{3}} \Psi_{3}\left(\theta_{0}, i_{1}, i_{2}, \tilde{l}_{3}\right) \mathrm{d} \tilde{\iota}_{3} \\
W_{\mathrm{m}}^{*}\left(\theta_{0}, \boldsymbol{i}\right) & =\int_{0}^{\boldsymbol{i}_{3}} \Psi_{3}\left(\theta_{0}, 0,0, \tilde{l}_{3}\right) \mathrm{d} \tilde{l}_{3} \\
& +\int_{0}^{\boldsymbol{i}_{1}} \Psi_{1}\left(\theta_{0}, \tilde{\iota}_{1}, 0, i_{3}\right) \mathrm{d} \tilde{l}_{1} \\
& +\int_{0}^{\boldsymbol{i}_{2}} \Psi_{2}\left(\theta_{0}, i_{1}, \tilde{l}_{2}, i_{3}\right) \mathrm{d} \tilde{l}_{2}
\end{aligned}
$$

The angle $\theta_{0}$ has to be varied to receive the coenergy over the whole domain of $\theta$.

The resulting torque can be calculated by the derivative of coenergy with respect to angle, as stated in (8). From a mathematical perspective, it makes sense to separate the terms of (13), (14) and (15) because they can be differentiated independently. Thus, equation (13) is separated to

$$
\begin{aligned}
W_{\mathrm{m}}^{*}(\theta, \boldsymbol{i}) & =W_{\mathrm{m}, 1}^{*}\left(\theta, i_{1}\right)+W_{\mathrm{m}, 2}^{*}\left(\theta, i_{1}, i_{2}\right) \\
& +W_{\mathrm{m}, 3}^{*}\left(\theta, i_{1}, i_{2}, i_{3}\right)
\end{aligned}
$$

And the coenergy derivatives follow to:

$$
\begin{aligned}
& T_{1}=\frac{\partial W_{\mathrm{m}, 1}^{*}}{\partial \theta} \\
& T_{2}=\frac{\partial W_{\mathrm{m}, 2}^{*}}{\partial \theta} \\
& T_{3}=\frac{\partial W_{\mathrm{m}, 3}^{*}}{\partial \theta}
\end{aligned}
$$

It is again pointed out, that these terms cannot be assigned generally to the individual phases. This is only admissible if there is no mutual coupling and thus the flux linkages of the different phases are independent of the other phase currents. By consideration of the mutual coupling the assignment of $T_{1}, T_{2}$ and $T_{3}$ is not unique as the comparison of (13) and (14) shows. This fact is important for the following implementation of the SRM model in Matlab Simscape.

\section{A. Implementation in Matlab Simscape}

Each motor phase is implemented in Matlab Simscape independently and with the same structure. The mutual coupling is only considered by the characteristic data and the input variables.

The following characteristic data in dependence of the respective motor angle and phase currents is stored in maps inside the SRM model:

$$
\begin{aligned}
& T_{\mathrm{a}}\left(\theta, i_{1}, i_{2}, i_{3}\right)=\frac{\partial}{\partial \theta} \int_{0}^{i_{1}} \Psi_{1}\left(\theta, \tilde{l}_{1}, i_{2}, 0\right) \cdot \mathrm{d} \tilde{l}_{1} \\
& T_{\mathrm{b}}\left(\theta, i_{1}, i_{2}, i_{3}\right)=\frac{\partial}{\partial \theta} \int_{0}^{i_{1}} \Psi_{1}\left(\theta, \tilde{\iota}_{1}, 0, i_{3}\right) \cdot \mathrm{d} \tilde{\iota}_{1}
\end{aligned}
$$

$$
\begin{aligned}
& L_{1}\left(\theta, i_{1}, i_{2}, i_{3}\right)=\frac{\partial \Psi_{1}\left(\theta, i_{1}, i_{2}, i_{3}\right)}{\partial i_{1}} \\
& L_{2}\left(\theta, i_{1}, i_{2}, i_{3}\right)=\frac{\partial \Psi_{1}\left(\theta, i_{1}, i_{2}, i_{3}\right)}{\partial i_{2}} \\
& L_{3}\left(\theta, i_{1}, i_{2}, i_{3}\right)=\frac{\partial \Psi_{1}\left(\theta, i_{1}, i_{2}, i_{3}\right)}{\partial i_{3}} \\
& \Lambda\left(\theta, i_{1}, i_{2}, i_{3}\right)=\frac{\partial \Psi_{1}\left(\theta, i_{1}, i_{2}, i_{3}\right)}{\partial \theta}
\end{aligned}
$$

whereas either $T_{\mathrm{a}}$ or $T_{\mathrm{b}}$ is used for the torque generation for a certain time step. The distinction between $T_{\mathrm{a}}$ or $T_{\mathrm{b}}$ is necessary because of symmetry reasons. In addition it can be used to describe long and short flux path behavior with only one data set [9]. According to (11) the characteristic maps $L_{1}, L_{2}, L_{3}$ and $\Lambda$ are used to calculate the phase voltage. The block diagram for one motor phase is depicted in Fig. 2.

This model structure is carried out analogously for each motor phase. Only the angle $\theta$ is electrically shifted by $120^{\circ}$ and the currents $i_{2}$ and $i_{3}$ are assigned to the other respective phase currents.

The implementation of the torque calculation is subject to certain restrictions, since not all terms of (13) to (15) are taken into account with $T_{\mathrm{a}}$ or $T_{\mathrm{b}}$. To explain this restriction and to demonstrate the advantages of this approach the concept of torque modelling is discussed in detail below.

\section{B. Torque Modelling}

Depending on the current waveform and the motor angle the resulting torque is generated by one, two or more phases. For the three phase SRM the torque is normally produced by the current of one or two phases see Fig. 3.

The region where two phases are active simultaneously is called the transition region. At this region one phase current is built up while the other phase current is reduced.

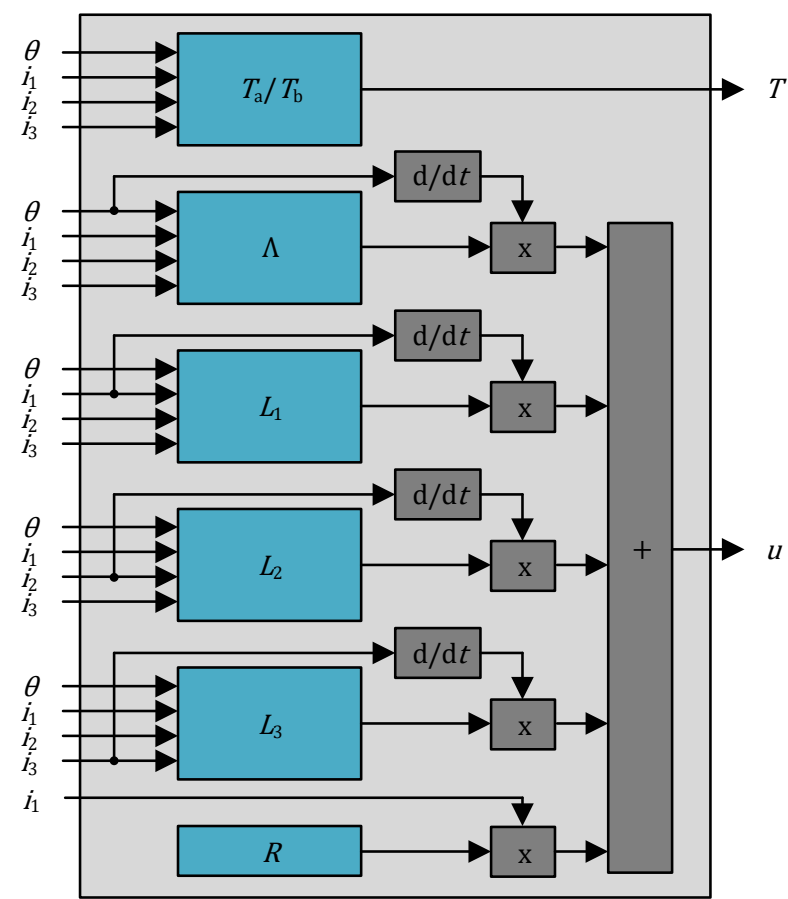

Fig. 2. Implementation of the SRM model in Matlab Simscape. This structure applies for each phase with the respective input variables. 


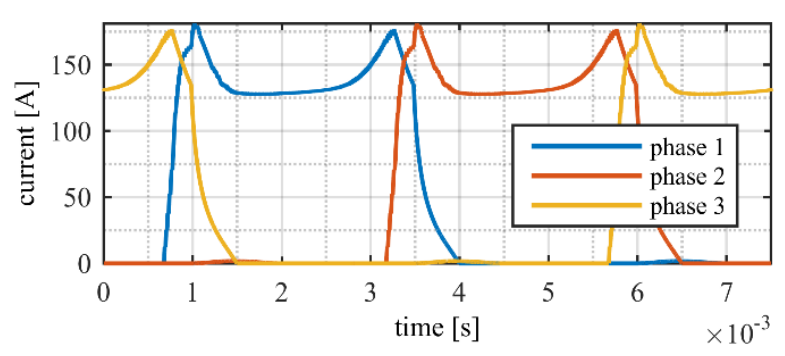

Fig. 3. Current waveform of the SRM in lower speed range.

Since every phase is modelled by the same structure and the same data set each motor phase has its own reference system, and thus its separate phase order. An example is given with respect to Fig. 4. Here the blue current profile of phase A reduces in the transition region and the red current of phase B builds up. The phase order is $(3-1-2)$ for both phases. Whereas phase A represents phase 1 in its reference system and phase $B$ also represents phase 1 in its reference system. Important is the fact that phase A passes through the transition $(3-1)$ and phase B goes through $(1-2)$.

If (20) is applied for both phases with its different instantaneous variables (Index A or B) and the resulting torque components are superimposed it follows:

$$
\begin{aligned}
T & =\frac{\partial}{\partial \theta} \int_{0}^{i_{1 . \mathrm{A}}} \Psi_{1}\left(\theta_{\mathrm{A}}, \tilde{l}_{1}, i_{2, \mathrm{~A}}, 0\right) \cdot \mathrm{d} \tilde{\imath}_{1} \\
& +\frac{\partial}{\partial \theta} \int_{0}^{i_{1 . \mathrm{B}}} \Psi_{1}\left(\theta_{\mathrm{B}}, \tilde{l}_{1}, i_{2, \mathrm{~B}}=0,0\right) \cdot \mathrm{d} \tilde{\imath}_{1}
\end{aligned}
$$

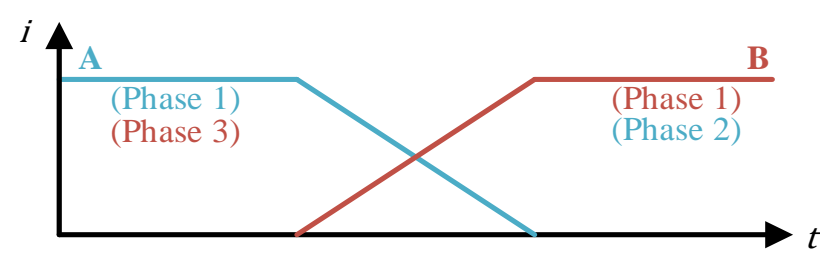

Fig. 4. Idealized transtition region of two phase currents. The current of phase B is built up and the current of phase A is reduced.

Comparison of (26) with (13) and its derivative with respect to motor angle shows that this is exactly the resulting torque of two active motor phases by taking into account mutual coupling. The application of (26) leads to the same results. The reason why a second torque map is necessary is given by symmetry conditions which will be discussed later.

At very high motor speeds it is possible that all three phases are active at the same time. The outgoing phase is normally very small which is why this influence can be neglected, due to low mutual coupling - see Fig. 5.

Only if the Continuous Conduction Mode [13] is applied, where phase current are not forced down to zero at the end of each period, the limits of the proposed model are reached.

On the other hand the model structure is very simple and the influence of the connection sequence can be modeled by only one dataset.

\section{Influence of the Phase Connecting Sequence on the Resulting Torque Generation}

The phases of an SRM are usually supplied independently by an inverter with bipolar voltage and unipolar current capability. Depending on the current direction or polarization of the different phase coils the mutual torque generation can be different. It can be distinguished between a short flux path (SFP) connection (see Fig. 6 left), where two consecutive stator teeth are oppositely polarized and a long flux path (LFP) connection (see Fig. 6 right), where two consecutive stator teeth are polarized in the same direction. While commutation the flux density in the main mutual flux path can be very high and thus saturation occurs. The longer the saturated flux paths are the higher is the total reluctance which lowers the maximum flux linkages.

The influence of an exemplary connection sequence can be seen in Fig. 7. The current waveforms are periodically but the resulting torque is not. The first and last transitions are done with a LFP connection and the middle transition is done with a SFP connection. Apparently the mutual torque generation with a LFP connection is much lower than with a SFP connection.

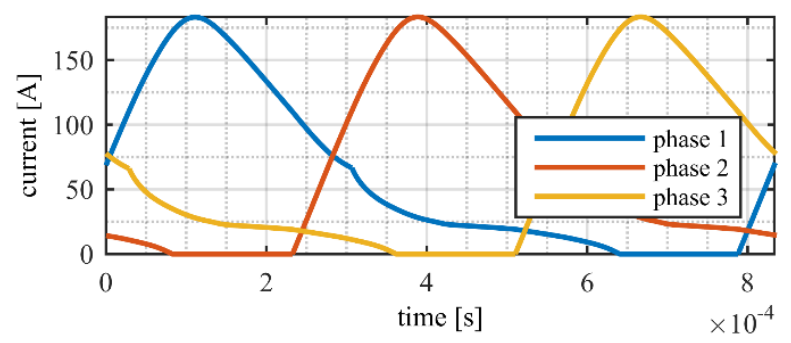

Fig. 5. Current waveform of the SRM at very high speed.

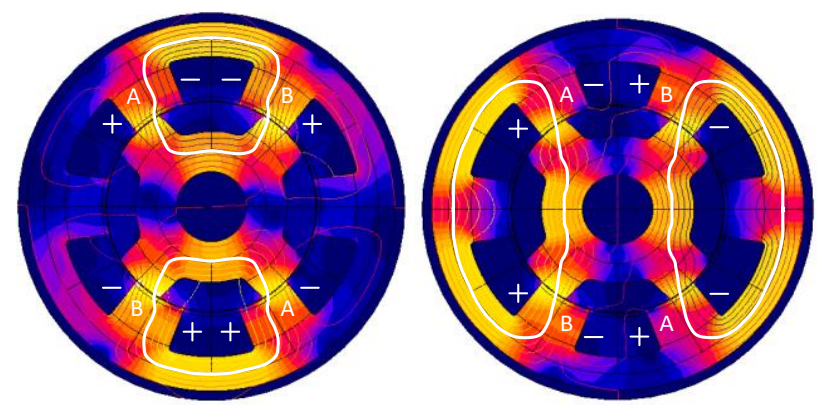

Fig. 6. Phase connection sequence with opposed polarization 'short flux path' (left) and with two adjacent poles polarized in the same direction 'long flux path' (right). Here the flux is commutating from pole pair A to pole pair $\mathrm{B}$.
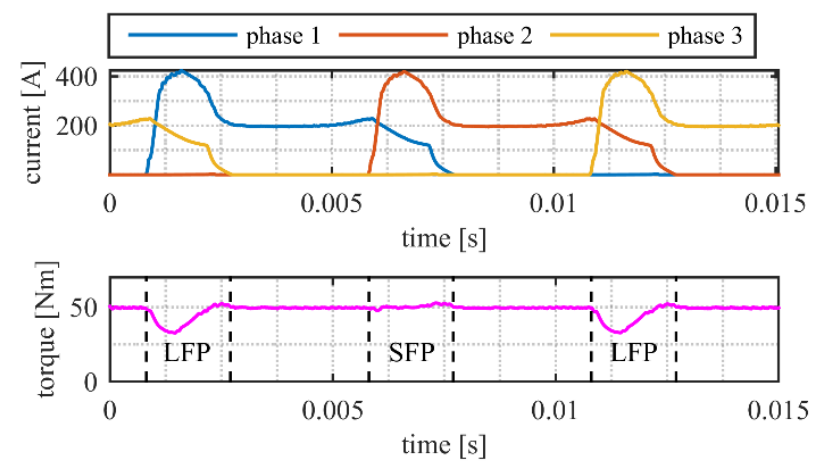

Fig. 7. Depending on the phase connection sequence the resulting motor torque is different for two overlapping phase currents. With LFP connection the resulting torque is much lower. 


\section{Data Acquisition and Processing}

The presented SRM model only requires the flux linkage in dependence of the motor angle and the phase currents. This can be obtained by measurement as explained in [3] or by finite element analysis (FEA). In this work the data acquisition is done by simulation with the program Flux2D from Cedrat [13].

For the data acquisition the rotor is fixed at a certain angle and through current sources the phase currents are systematically varied to get the corresponding flux linkages. After that the rotor is moved by an angle step and the procedure is repeated until a whole electrical period is recorded. If only the behavior of LFP or SFP connection is of interest, half an electrical period is sufficient because of symmetry conditions.

The data processing and the consideration of symmetry conditions is explained in detail in the related conference paper [9].

\section{E. Modelling of Short and Long Flux Path Behavior with One Data Set}

As stated before both the SFP and LFP behavior can be modeled very accurately with only one data set. This can be done if either phase 2 or phase 3 is connected in SFP connection and the other in LFP connection while the data is collected. In this case LFP and SFP behavior can be switched by interchanging $T_{\mathrm{a}}$ and $T_{\mathrm{b}}$ in (22) and (23).

This method causes a small error for $\theta \in\left(180^{\circ}, 360^{\circ}\right]$ because the wrong 'flux path' connection is taken into account for this range. Since the main transition range is completed before $\theta \in\left(180^{\circ}, 360^{\circ} \mathrm{]}\right.$, this has only a small influence on the turn off voltage but torque calculation has almost no errors.

The application of this methodology is very useful if a SRM should be operated with a direct torque control method which should be able to consider both SFP and LFP behavior. Such an example will be shown in the following Section.

\section{VALIDATION OF THE MODEL}

For the validation of the model, it is applied to a three phase SRM with 12 stator teeth and 8 rotor teeth. The motor has been designed by a cascaded design methodology which optimizes the geometry by an outer geometry optimization and an inner control optimization loop for the application in an electric vehicle as introduced in [14]. An overview of the motor parameters is given in Table I.

The flux linkage recording of phase 1 in dependence of all motor currents and the motor angle (from $0^{\circ}$ to $180^{\circ}$ ) is done by a static FEA with the program Flux $2 D$. From that the parameter maps are determined as explained in the Sections before. For the following examples 25 angle grid points and only 15 grid points for each phase current ranging from $0 \mathrm{~A}$ to $350 \mathrm{~A}$ have been chosen which make still 84,375 grid points in total. With an Intel i7-377K and 16 GB RAM this takes about 72 hours of calculation time.

For the validation of the model the current profiles are calculated offline in Matlab Simscape and are then imported into a dynamic FEA in Flux $2 D$ by table values. To calculate the current profiles the model is implemented in Matlab Simscape as depicted in Fig. 2 in combination with an inverter model. The operation is carried out with a Direct Instantaneous Torque Control (DITC) scheme [15] with the torque model described in this work in Section III.

As a first example an operating point with 1000 revolutions per minute and a target torque of $80 \mathrm{Nm}$ is shown in Fig. 8. Here both the phase connection sequence and the data recording are done with a SFP connection.

The resulting torque in the FEA has only a small ripple due to a limited switching frequency and the maximum voltage is mostly between $+780 \mathrm{~V}$ and $-780 \mathrm{~V}$ which indicates that the Simscape model is very accurate. The small voltage deviation at $1.25 \mathrm{~ms}, 3.75 \mathrm{~ms}$ and $6.25 \mathrm{~ms}$ can be further reduced by a higher number of current grid points but this further increases the calculation or measurement effort of the data acquisition. In comparison to this Fig. 9 shows the resulting torque without taking into account mutual coupling.

In Section III.E it is described how both SFP and LFP behavior can be modeled with one dataset. Application of this method and interchanging between $T_{\mathrm{a}}$ and $T_{\mathrm{b}}$ whether SFP or LFP mutual coupling is presented, leads to the result in Fig. 10.

TABLE I.

MOTOR PARAMETERS

\begin{tabular}{|c|c|}
\hline Property & Value \\
\hline rated power & $150 \mathrm{~kW}$ \\
\hline maximum speed & $30,000 \mathrm{rpm}$ \\
\hline active length & $205 \mathrm{~mm}$ \\
\hline stator diameter & $170 \mathrm{~mm}$ \\
\hline dc link voltage & $780 \mathrm{~V}$ \\
\hline dc link current & $300 \mathrm{~A}$ \\
\hline
\end{tabular}
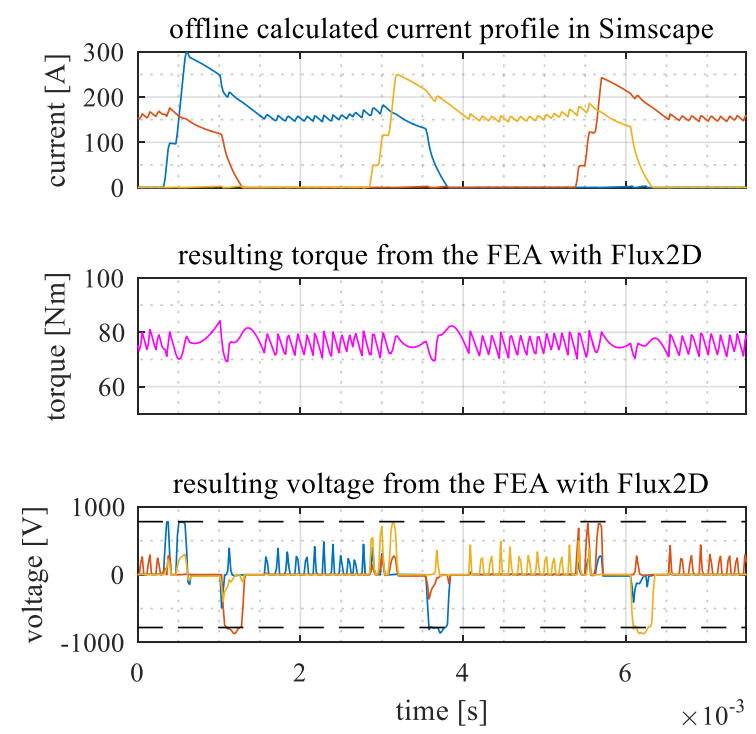

Fig. 8. Resulting torque and voltage in the FEA with respect to an offline calculated current profile. (blue: phase 1; yellow: phase 2; red: phase 3) 

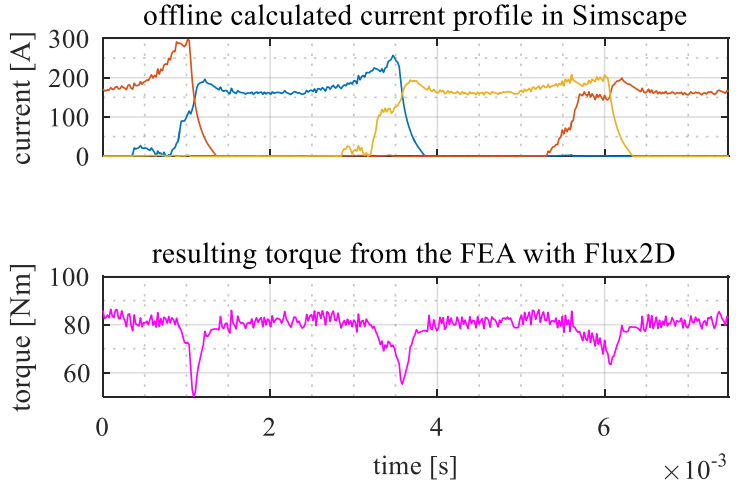

Fig. 9. Resulting torque in the FEA with respect to an offline calculated current profile without taking into account mutual coupling. (blue: phase 1; yellow: phase 2; red: phase 3)
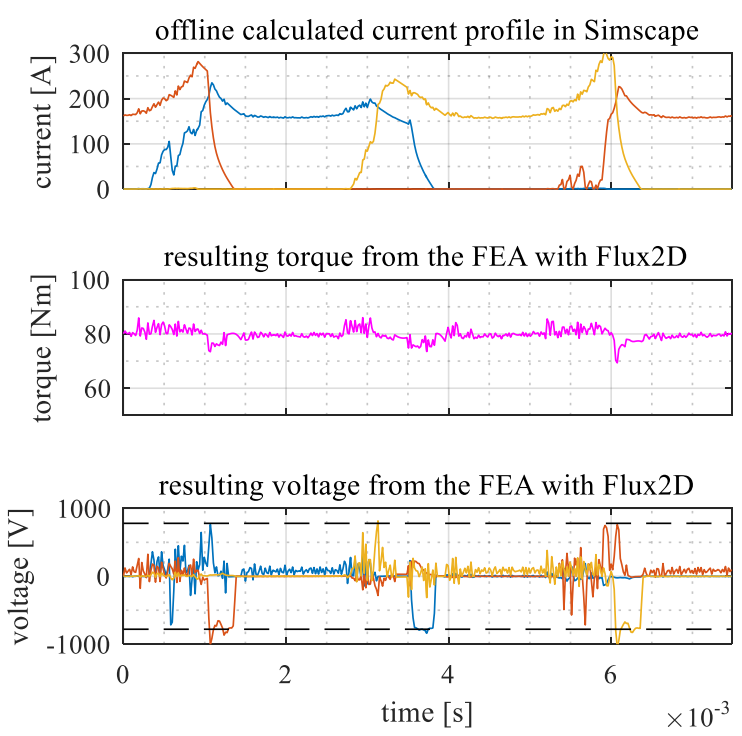

Fig. 10. Resulting torque and voltage in the FEA with respect to an offline calculated current profile with changing SFP and LFP transition regions. (blue: phase 1; yellow: phase 2; red: phase 3)

Fig. 10 shows that the torque calculation is very good but the voltage has a very little error. Nonetheless, in direct torque control methods only torque calculation is of interest and thus the suggested model can be used without restrictions.

\section{EXPERIMENTAL VERIFICATION}

In order to verify the presented model, measurements on a SRM prototype are carried out and compared to the simulation results of the Matlab Simscape model. Average Torque Control [18] is used as a control strategy setting the active phase currents to a constant level by a hysteresis controller. Target current and switching angles are optimized offline in advance and stored in look-up tables.

Phase currents are measured at different speeds both in SFP and LFP configuration. A reduced DC voltage of $100 \mathrm{~V}$ is used to supply the inverter. In Fig. 11 and Fig. 12 the results are shown for two different operating points at middle and high motor speed. Due to a higher inductance the phase current in LFP configuration is built up more slowly. This behavior is shown accurately in the presented model. Only in the region of current reduction in LFP configuration relevant deviations can be seen.
Furthermore, the average torque is measured and compared to the simulation results. The results for the two operating points can be seen in Table II and Table III.

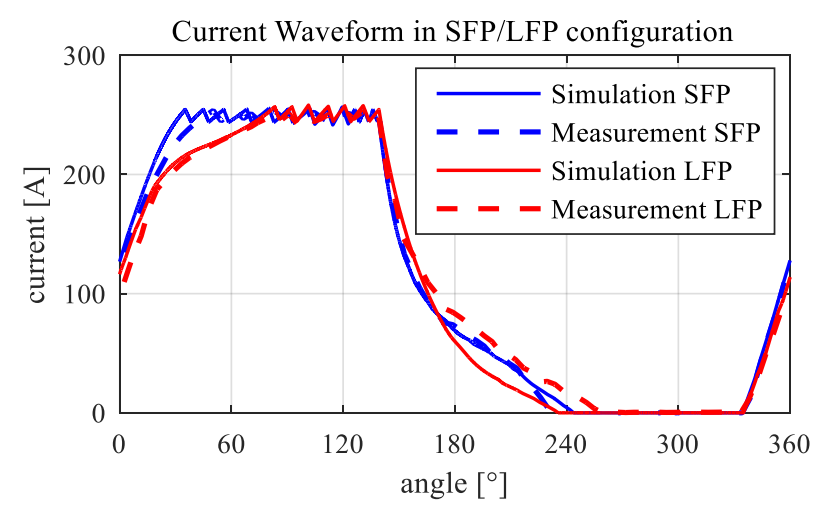

Fig. 11. Simulated and measured current waveforms at $900 \mathrm{rpm}$ and a target torque of $115 \mathrm{Nm}$.

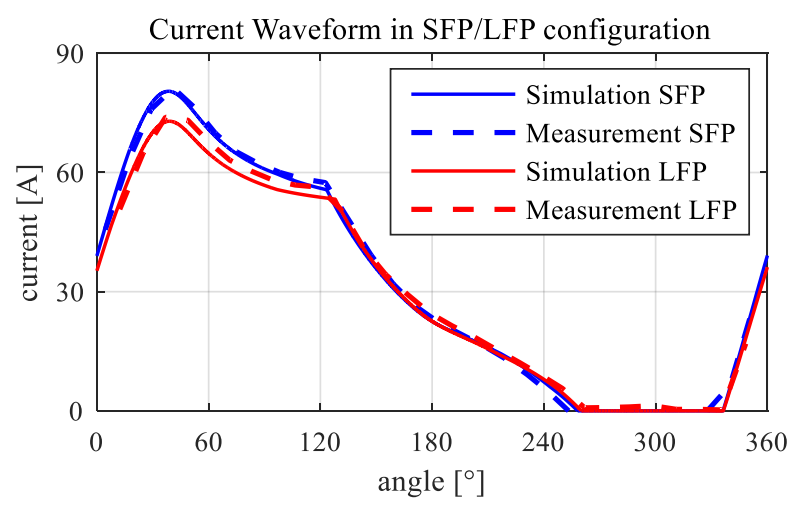

Fig. 12. Simulated and measured current waveforms at $2500 \mathrm{rpm}$ and a target torque of $16 \mathrm{Nm}$.

TABLE II.

Simulated AND MEASURED TORQUE AT 900 RPM AND A TARGET TORQUE OF $115 \mathrm{NM}$

\begin{tabular}{|l|c|c|}
\cline { 2 - 3 } \multicolumn{1}{c|}{} & SFP & LFP \\
\hline simulation & $117.0 \mathrm{Nm}$ & $112.9 \mathrm{Nm}$ \\
\hline measurement & $109.3 \mathrm{Nm}$ & $102.7 \mathrm{Nm}$ \\
\hline deviation & $+7 \%$ & $+9.9 \%$ \\
\hline
\end{tabular}

TABLE III.

Simulated AND MEASURED TORQUE AT 2500 RPM AND A TARGET TORQUE OF 16 NM

\begin{tabular}{|l|c|c|}
\cline { 2 - 3 } \multicolumn{1}{c|}{} & SFP & LFP \\
\hline simulation & $15.8 \mathrm{Nm}$ & $14.5 \mathrm{Nm}$ \\
\hline measurement & $15.0 \mathrm{Nm}$ & $13.5 \mathrm{Nm}$ \\
\hline deviation & $+5.3 \%$ & $+7.4 \%$ \\
\hline
\end{tabular}

As expected the measured torque in SFP mode is higher than in LFP mode. In the first operating point, measured torque in SFP configuration is $6.4 \%$ higher and in the second operating point $11.1 \%$ higher than in LFP configuration.

In both cases the simulated torques are higher than the measured values. As outlined before the simulation model only includes copper losses neglecting other losses such as iron or mechanical losses. Consequently the simulated torque needs to be higher. 
The same result is presented in Fig. 13 showing the maximum torque of the SRM and the corresponding efficiency in these operating points. At higher speeds the difference between the simulated and the measured efficiency increases. This indicates that frequency dependent eddy current losses and iron losses need to be considered.

\section{CONCLUSION}

In this work the general mathematical model of the switched reluctance machine has been derived by the aid of energy functionals. The system behavior can be completely derived by the flux linkage in dependence of the state variables, which are the motor angle and the phase currents.

The model has been applied to a three phase SRM. The proposed model implementation offers the opportunity to approximate the system behavior of both short and long flux path behavior with only one model structure and data set.

The validation is done by importing offline calculated current profiles into a finite element analysis and comparing the resulting torque and voltage with the target values. It is shown that the model has only a small deviation which can be further reduced by a larger resolution of the recorded flux linkage map.

In the last Section the model is verified by measurement results. The high correlation between the measured and simulated current profiles prove a high accuracy of the introduced model. However, for an accurate torque modelling, the consideration of frequency dependent losses is necessary, which will be part of the following research.
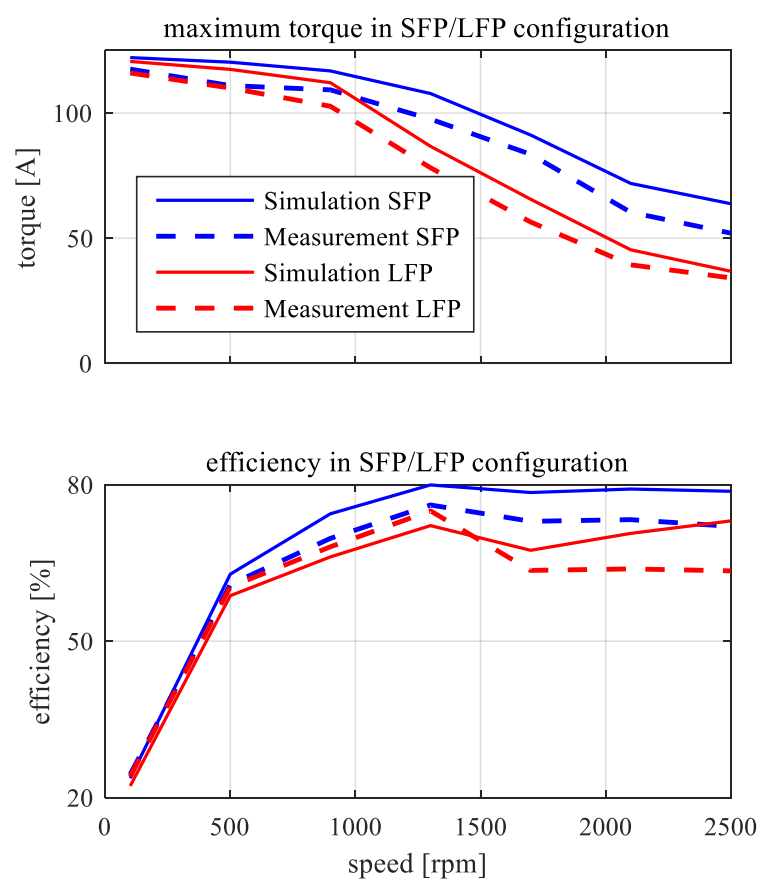

Fig. 13. Simulated and measured maximum torque and corresponding efficiency with SFP and LFP configuration.

\section{REFERENCES}

[1] T. J. E. Miller, Switched Reluctance Motors and Their Controls: Magna Physics Publ., 1993.

[2] P. Debiprasad and V. Ramanarayanan, "Mutual Coupling and Its Effect on Steady-State Performance and Position Estimation of Even and Odd Number Phase Switched Reluctance Motor Drive," Magnetics, IEEE Transactions on, vol. 43, pp. 3445-3456, 2007. http://dx.doi.org/10.1109/TMAG.2007.898101

[3] Q. Bingni, S. Jiancheng, L. Tao, and Z. Hongda, "Mutual coupling and its effect on torque waveform of even number phase switched reluctance motor," in Electrical Machines and Systems, 2008. ICEMS 2008. International Conference on, 2008, pp. 3405-3410.

[4] Y. Jin, B. Bilgin, and A. Emadi, "Elimination of Mutual Flux Effect on Rotor Position Estimation of Switched Reluctance Motor Drives," Power Electronics, IEEE Transactions on, vol. 30, pp. 1499-1512, 2015.

http://dx.doi.org/10.1109/TPEL.2014.2319238

[5] A. Fehn, Modellierung und Regelung von Stellantrieben mit geschalteten Reluktanzmotoren: VDI-Verlag, 2005.

[6] P. Debiprasad and V. Ramanarayanan, "An accurate position estimation method for switched reluctance motor drive," in Power Electronic Drives and Energy Systems for Industrial Growth, 1998. Proceedings. 1998 International Conference on, 1998, pp. 523-528 Vol. 2.

[7] B. Han-Kyung and R. Krishnan, "A novel approach to control of switched reluctance motors considering mutual inductance," in Industrial Electronics Society, 2000. IECON 2000. 26th Annual Confjerence of the IEEE, 2000, pp. 369-374 vol.1. http://dx.doi.org/10.1109/iecon.2000.973178

[8] R. B. Inderka and R. W. De Doncker, "DITC-direct instantaneous torque control of switched reluctance drives," in Industry Applications Conference, 2002. 37th IAS Annual Meeting. Conference Record of the, 2002, pp. 1605-1609 vol.3. http://dx.doi.org/10.1109/ias.2002.1043749

[9] M. Greule and M. Doppelbauer, "Modelling of High Torque Density Switched Reluctance Motors with Mutual Coupling," in International Conference on Electrical Drives and Power Electronics (EDPE), Tatranská Lomnica, Slovakia, 2015. http://dx.doi.org/10.1109/EDPE.2015.7325293

[10] C. B. Arnau, A. D. Cerezo, and U. P. d. C. I. d. O. i. C. d. S. Industrials, Modeling and control of electromechanical systems: Institut d'Organització i Control de Sistemes Industrials, 2005.

[11] R. C. Stevenson, "The role of coenergy \& the development of a comprehensive analytical model for a PM motor," in Vehicle Power and Propulsion Conference, 2009. VPPC '09. IEEE, 2009, pp. 275-282.

http://dx.doi.org/10.1109/vppc.2009.5289841

[12] I. N. Bronstein, H. Mühlig, G. Musiol, and K. A. Semendjajew, Taschenbuch der Mathematik: Europa Lehrmittel Verlag, 2013.

[13] H. Hannoun, M. Hilairet, and C. Marchand, "Experimental Validation of a Switched Reluctance Machine Operating in Continuous-Conduction Mode," Vehicular Technology, IEEE Transactions on, vol. 60, pp. 1453-1460, 2011. http://dx.doi.org/10.1109/TVT.2011.2124478

[14] M. Greule and M. Doppelbauer, "Cascaded Design Methodology for Switched Reluctance Motors Considering Optimum Control," presented at the International Electric Machines and Drives Conference (IEMDC), Coeur D'Alene, Idaho, 2015. http://dx.doi.org/10.1109/iemdc.2015.7409285

[15] R. B. Inderka and R. W. A. A. De Doncker, "DITC-direct instantaneous torque control of switched reluctance drives," Industry Applications, IEEE Transactions on, vol. 39, pp. 1046$1051,2003$. http://dx.doi.org/10.1109/TIA.2003.814578

[16] M. Greule, M. Boxriker, B. Zhang, and M. Doppelbauer, "Numerical Iron Loss Computation Method for Switched Reluctance Motors," unpublished.

[17] C. Carstensen, "Eddy Currents in Windings of Switched Reluctance Machines," Dr.-Ing., Fakultät für Elektrotechnik und Informationstechnik, Rheinisch-Westf ' alischen Technischen Hochschule Aachen, 2008.

[18] R. Krishnan, Switched Reluctance Motor Drives: Modeling, Simulation, Analysis, Design, and Applications: Taylor \& Francis, 2010. 